\title{
ON CERTAIN THEOREMS OF PÓLYA AND BERNSTEIN
}

\section{BY NORMAN LEVINSON*}

1. Introduction. As a generalization of the Fabry gap theorem Pólya $\uparrow$ has proved the following result.

THEOREM 1. The function $f(z)$ given by the power series

$$
f(z)=\sum_{0}^{\infty} a_{n} z^{m_{n}}
$$

with

$$
\lim _{n \rightarrow \infty} \frac{n}{m_{n}}=D
$$

has $\ddagger$ at least one singularity on every arc on its circle of convergence whose intercepted angle exceeds $2 \pi D$.

$\mathrm{V}$. Bernstein§ has proved the following theorem.

Theorem 2. Let $\phi(z)$ be analytic in some sector $|\mathrm{am} z| \leqq \alpha$. Let

$$
\varlimsup_{r \rightarrow \infty} \frac{\log |\phi(r)|}{r}=a .
$$

Suppose there exists some $b$ such that for any $\epsilon>0$,

$$
\phi\left(r e^{i \theta}\right)=O\left(e^{(a \cos \theta+b|\sin \theta|+\epsilon) r}\right), \quad(|\theta| \leqq \alpha) .
$$

Let $\left\{\lambda_{n}\right\}$ be an increasing sequence of positive numbers such that

$$
\lim _{n \rightarrow \infty} \frac{n}{\lambda_{n}}=D, \quad \quad \lambda_{n+1}-\lambda_{n} \geqq d>0 .
$$

* National Research Fellow.

$\dagger$ Pólya, Untersuchungen über Lücken und Singularitäten von Potenzreihen, Mathematische Zeitschrift, vol. 29 (1929).

$\ddagger$ The theorem with (2) replaced by the Pólya maximal density is obviously an immediate corollary of Theorem 1.

$\S$ Bernstein, Séries de Dirichlet, 1933, Chap. 9. 
If $\pi D>b$, then

$$
\varlimsup_{n \rightarrow \infty} \frac{\log \left|\phi\left(\lambda_{n}\right)\right|}{\lambda_{n}}=a=\varlimsup_{r \rightarrow \infty} \frac{\log |\phi(r)|}{r} .
$$

Bernstein uses rather deep results from the theory of Dirichlet series in proving this theorem and points out that as yet no proof has been given using ordinary function theory. Here we will give a simple function theoretic proof of this theorem and then show how it leads at once to a proof of Theorem 1. We will then discuss other theorems of Pólya and Bernstein.

2. Proofs of Theorems 1 and 2. We require the following results of Pólya* and Carlson.

Let $\left\{\lambda_{n}\right\}$ satisfy (5). If

$$
F(z)=\prod_{1}^{\infty}\left(1-\frac{z^{2}}{\lambda_{n}^{2}}\right),
$$

then

$$
\begin{aligned}
& \lim _{r \rightarrow \infty} \frac{\log \left|F\left(r e^{i \theta}\right)\right|}{r}=\pi D \sin |\theta|, \quad(\theta \neq 0, \pi) . \\
& \varlimsup_{x \rightarrow \infty} \frac{\log |F( \pm x)|}{x}=0 .
\end{aligned}
$$

To any $\epsilon>0$ there exists an $A$ and a $B$ such that

$$
|F(z)|>A e^{-\epsilon|z|}, \quad\left|z \pm \lambda_{n}\right| \geqq \frac{1}{2} d,
$$

and

$$
\left|F^{\prime}\left(\lambda_{n}\right)\right|>B e^{-\epsilon \lambda_{n}} .
$$

Proof of Theorem 2. Let us suppose that $\pi D>b$ but that contrary to $(6)$ we have

$$
\varlimsup_{n \rightarrow \infty} \frac{\log \left|\phi\left(\lambda_{n}\right)\right|}{\lambda_{n}}<c<a .
$$

From (9) and (10)

* For a proof of these results see Pólya, loc. cit., p. 568, Theorem XI and the two examples following it. 


$$
g(z)=\sum_{1}^{\infty} \frac{\phi\left(\lambda_{n}\right) e^{-c \lambda_{n}}}{F^{\prime}\left(\lambda_{n}\right)\left(z-\lambda_{n}\right)} e^{c z} F(z)
$$

is an entire function. Since $g\left(\lambda_{n}\right)=\phi\left(\lambda_{n}\right)$, it follows that

$$
\psi(z)=\frac{\phi(z)-g(z)}{F(z)}
$$

is analytic for $\mid$ am $z \mid \leqq \alpha$. From (4), (8), and (11), it follows that there exists a $B>0$ such that, for $\left|z-\lambda_{n}\right| \geqq d / 2$,

$$
\psi(z)=O\left(e^{B|z|}\right) .
$$

Since $\psi(z)$ is analytic (13) must be true even when $\left|z-\lambda_{n}\right|<d / 2$.

We assume $\alpha<\pi / 2$, which is obviously no restriction. If we set $(\pi D-b) \tan \alpha=\gamma$, then (4), (7), (11), and (12) give us

$$
\psi\left(r e^{ \pm i \alpha}\right)=O\left(e^{r \cos \alpha(a-\gamma+\epsilon \sec \alpha)}+e^{c r \cos \alpha}\right) .
$$

If we now choose $\epsilon<\gamma \cos \alpha / 2$, it is clear that

$$
\psi\left(r e^{ \pm i \alpha}\right)=O\left(e^{r p \cos \alpha}\right), \quad p=\max (a-\gamma / 2, c) .
$$

That is, $e^{-p z} \psi(z)$ is bounded on am $z= \pm \alpha$. But by a well known theorem of Phragmén-Lindelöf this and (13) imply that it is bounded in the entire sector $\mid$ am $z \mid \leqq \alpha<\pi / 2$. Thus, in particular,

$$
\psi(x)=O\left(e^{p x}\right), \quad p=\max (a-\gamma / 2, c) .
$$

By (12), $\phi(x)=F(x) \psi(x)+g(x)$. Thus using (7), (11), and (14), we find

$$
\varlimsup_{x \rightarrow \infty} \frac{\log |\phi(x)|}{x} \leqq \max (a-\gamma / 2, c),
$$

contrary to (3). This proves the theorem.

ProOF of THEOREM 1. We suppose without restriction that the radius of convergence is 1 . Suppose there exists an arc of the unit circle with intercepted angle $2 \pi \beta>2 \pi D$ on which $f(z)$ is analytic. We can assume that the arc contains the point $z=-1$ and is symmetric about this point. We define $C$ as the curve consisting of the line from $(1+\delta) e^{-\pi i(1-\beta)}$ to $e^{-\pi i(1-\beta)} / 2$, the arc of the circle $|z|=1 / 2$ from $e^{-\pi i(1-\beta)} / 2$ to $e^{\pi i(1-\beta)} / 2$ in the positive sense, and the line from $e^{\pi i(1-\beta)} / 2$ to $(1+\delta) e^{\pi i(1-\beta)}$. We define $J$ 
as the curve along the arc of the circle $|z|=1+\delta$ from the point $(1+\delta) e^{\pi i(1-\beta)}$ to the point $(1+\delta) e^{-\pi i(1-\beta)}$ in the positive sense.

We take $\delta>0$ but so small that $f(z)$ is analytic on the closed curve $C+J$ and inside the region bounded by this curve.

Let $\left\{\mu_{n}\right\}$ be the positive integers complementary to the $\left\{m_{n}\right\}$. Then clearly

$$
\lim _{n \rightarrow \infty} \frac{n}{\mu_{n}}=1-D .
$$

Since the coefficients of $z^{\mu_{n}}$ in the power series of $f(z)$ are zero,

$$
\int_{C} \frac{f(z)}{z^{\mu_{n}+1}} d z+\int_{J} \frac{f(z)}{z^{\mu_{n}+1}} d z=0 .
$$

Since $f(z)$ is bounded on $J$, (16) gives

$$
\int_{C} \frac{f(z)}{z^{\mu_{n}+1}} d z=O\left(\frac{1}{(1+\delta)^{\mu_{n}}}\right)
$$

Let

$$
H(w)=\frac{1}{2 \pi i} \int_{C} \frac{f(z)}{z^{w+1}} d z .
$$

Writing $z^{-w}$ as $r^{-w} e^{-i w \theta}$, we see that

$$
\left|z^{-u-i v}\right| \leqq r^{-u} e^{\pi|v|(1-\beta)}
$$

on $C$. Therefore for $\delta<1$,

$$
H(u+i v)=O\left(2^{|u|} e^{\pi|v|(1-\beta)}\right) .
$$

Let

$$
\lim _{u \rightarrow \infty} \frac{\log |H(u)|}{u}=a
$$

Then by (19),

$$
H(w) e^{-(a+\epsilon) w+\pi i(1-\beta) w}
$$

is bounded along the positive imaginary axis and by (20) with any $\epsilon>0$, it is bounded along the positive real axis. Thus by a well known theorem of Phragmén and Lindelö it is bounded in the first quadrant. That is, in the first quadrant 


$$
H(w)=O\left(e^{(a+\epsilon) u+\pi(1-\beta)|v|}\right),
$$

Similarly this result is true in the fourth quadrant and therefore for all $u \geqq 0$. From (17) with $\delta<1$,

$$
H\left(\mu_{n}\right)=O\left((1+\delta)^{-\mu_{n}}\right)=O\left(e^{-\delta \mu_{n} / 2}\right) .
$$

We can now apply Theorem 1 to $H(w)$ using (20), (21), (22), and (15). The fact that $\pi(1-D)>\pi(1-\beta)$ shows that $a \leqq-\delta / 2$. That is,

$$
H(u)=O\left(e^{-u \delta / 4}\right), \quad(u>0) .
$$

Thus

$$
\begin{aligned}
a_{n} & =H\left(\lambda_{n}\right)+\frac{1}{2 \pi i} \int_{J} \frac{f(z)}{z^{\lambda_{n}+1}} d z \\
& =H\left(\lambda_{n}\right)+O\left(\frac{1}{(1+\delta)^{\lambda_{n}}}\right)=O\left(e^{-\delta \lambda_{n} / 4}\right) .
\end{aligned}
$$

Therefore the radius of convergence exceeds 1 , contrary to our assumption. This proves the theorem.

3. Extended Results. We can get all the theorems of Bernstein of the same type as Theorem 2 which are given in Chapter 9 of his Séries de Dirichlet as easily as Theorem 2. Moreover, we can replace the real sequence $\left\{m_{n}\right\}$ by a complex sequence $\left\{z_{n}\right\}$, where $\mid$ am $z_{n} \mid \rightarrow 0$ as $n \rightarrow \infty$. It is also possible to obtain certain new theorems of a similar nature but considerably more precise.

As regards Theorem 1, the method used will also yield the gap theorems of Pólya on entire functions. Here we require a more precise statement of Theorem 2 which is however no harder to prove. We will consider these various results in some detail in the near future.

Princeton University 